\title{
Comparison of Nasal and Frontal BIS Monitoring in Neurosurgery: Does the Site of Sensor Placement Affect the BIS Values?
}

\author{
Konul Hajiyeva1, Basak Ceyda Meco², Cigdem Yildirim Guclu², Dilek Yorukoglu², \\ Beyza Doganay3, Mehmet Oral ${ }^{2}$ \\ ${ }^{1}$ Memorial Hospital Ankara, Ankara, Turkey \\ ${ }^{2}$ Ankara University School of Medicine, Department of Anesthesiology and Reanimation, Ankara, Turkey \\ ${ }^{3}$ Ankara University School of Medicine, Department of Biostatistics, Ankara, Turkey \\ Email: konul.hadschieva@gmail.com
}

How to cite this paper: Hajiyeva, K., Meco, B.C., Guclu, C.Y., Yorukoglu, D., Doganay, B. andOral, M. (2021) Comparison of Nasal and Frontal BIS Monitoring in Neurosurgery: Does the Site of Sensor Placement Affect the BIS Values? International Journal of Clinical Medicine, 12, 108-114.

https://doi.org/10.4236/ijcm.2021.123012

Received: February 23, 2021

Accepted: March 19, 2021

Published: March 22, 2021

Copyright $\odot 2021$ by author(s) and Scientific Research Publishing Inc. This work is licensed under the Creative Commons Attribution International License (CC BY 4.0).

http://creativecommons.org/licenses/by/4.0/

\begin{abstract}
Background and Goal of Study: Intraoperative awareness is a serious but preventable complication of general anaesthesia. Bispectral index (BIS) is the most widely used method monitoring anaesthesia depth. BIS monitoring requires attachment of forehead sensors, which poses a challenge when the surgical field involves the forehead. We aimed to compare the gold standard forehead position of BIS sensors with an alternative position across the nasal dorsum for neurosurgical procedures. Materials and Methods: After ethical committee approval and informed consent were obtained, 62 patients were enrolled in this prospective observational study. Frontal and nasal BIS values were compared in all patients. Results and Discussion: The mean BIS value from frontal versus nasal sensors was $49 \pm 22$ and $49 \pm 21$ respectively (n: 62). These values were statistically correlated (ICC $0.78, \mathrm{p}<0.001$ ) indicating that nasal BIS measurement does not present a disadvantage for routine use when needed. Conclusion: Our data reveal that for measuring anesthesia depth, BIS sensor placement on the nasal dorsum shows comparable efficiency in comparison to standard frontal measurements
\end{abstract}

\section{Keywords \\ BIS, Neuromonitoring, Neurosurgery}

\section{Introduction}

Intraoperative awareness, with or without recall, continues to be a topic of clinical significance and neurophysiological interest [1]. The unintended experience 
and memory of surgical or procedural events can be devastating for patients and remains an active area of study. Intraoperative awareness also has consequences for the anesthetist. A recent examination by the American Society of Anesthesiologists' (ASA) Closed Claim Project revealed that 2\% of all claims were for awareness [2] Such claims are frequently successful, and poor anesthetic technique is often blamed. Hence, monitoring the depth of anesthesia has become increasingly necessary. Bispectral index (BIS) monitoring is a useful adjunct to monitoring the depth of anesthesia and reducing the risk of awareness for high-risk groups. BIS-guided anaesthesia compared to clinical signs may reduce the risk of intraoperative awareness and improve early recovery times in people undergoing surgery under general anaesthesia [3]. A frontotemporal placement of electrodes is now considered to be the gold standard for BIS monitoring [4]. However, in some neurosurgical cases the surgical incision site may compromise this placement. Here, we aimed to compare the gold standard forehead position of BIS sensors with an alternative position across the nasal dorsum for neurosurgical procedures.

\section{Methods}

After ethical committee approval (Ankara University School of Medicine on October 21, 2016) informed consent was obtained giving adequate information concerning the study, providing adequate opportunity for the patient to consider all options, responding to the patient's questions, ensuring that the patient has comprehended this information, obtaining patient's voluntary agreement. 62 patients who were scheduled for an elective operation under general anesthesia at the neurosurgical unit in Ankara University School of Medicine were enrolled in this prospective observational study. The inclusion criteria were ASA I-III patients between 18 - 80 years of age undergoing elective neurosurgical operations with no contraindications for the placement of electrodes (BISTM Quatro Sensors, Aspect Medical Systems, Newton, MA, USA) over the forehead and nasal dorsum (e.g., the boundary area being too close to the surgical site or having skin infections). Patients with disabling central nervous system or cerebrovascular disease, those currently taking psychiatric medication, and those with a history of neurosurgical intervention were excluded. Standard monitoring was performed upon arrival in the operating room (non-invasive blood pressure measurements, electrocardiography, oxygen saturation, and TOF).

Statistical Analysis: The sample size was calculated using equivalence testing and Bland-Altman analysis. Numerical data were summarized as mean \pm standard deviation and median (minimum-maximum), whereas frequencies and percentages were used for categorical data. Consistency between frontal and nasal measurements was evaluated with intraclass correlation coefficient (ICC). When consistency between multiple repeated measurements were considered, a three level linear mixed effects model was fitted taking BIS values as dependent variable and patients, repeated measurements nested within frontal/nasal were 
taken as random effects. Variance components then used to calculate ICC. Bootstrap method with 10,000 samples was used to obtain $95 \%$ confidence intervals (CI). Based on the guideline given by Koo and Li (2016), ICC values below 0.5 considered as poor, 0.50 to 0.75 as moderate, 0.75 to 0.90 as good, and above 0.90 as excellent. For all statistical calculations IBM SPSS Statistics version 20.0 and $\mathrm{R}$ version 4.0.0. (package rmcorr) were used and $\mathrm{p}$ value $<0.05$ is accepted statistical significant [5].

Before the induction of anesthesia, two BIS sensors (BIS ${ }^{\mathrm{TM}}$ Quatro Sensors, Aspect Medical Systems, Newton, MA, USA) were adhered to each patient: one across the forehead and the other across the nasal bridge. Each sensor was attached to its own BIS monitor (BIS-Vista ${ }^{\mathrm{TM}}$ monitors, Aspect Medical Systems, Newton, MA, USA). Nasal sensors were placed on the same side of the face with circle 1 on the nasal dorsum, circle 2 on the nasofacial angle, circle 4 on zygomatic bone, and circle 3 on the ipsilateral temporal area (Figure 1).

Before induction of anesthesia, all patients were sedated with $1 \mathrm{mcg} / \mathrm{kg}$ fentanyl. Anesthesia was induced with $2.5 \mathrm{mg} / \mathrm{kg}$ propofol, and $1 \mathrm{mg} / \mathrm{kg}$ of rocuronium was administered as a muscle relaxant. Anesthesia was then maintained with $100-200 \mathrm{mcg} / \mathrm{kg} / \mathrm{min}$ propofol and $0.25-1 \mathrm{mcg} / \mathrm{kg} / \mathrm{min}$ remifentanil with a target frontal BIS value of $40-60$. During the procedure, BIS values were collected from the two different positions before the induction of anesthesia, at loss of the eyelash reflex, after intubation, after the first surgical incision, every 15 minutes during the intraoperative period, and at spontaneous eye opening upon emergence from anesthesia.

The TIVA infusion was stopped after skin closure, and sugammadex (dose was according to the TOF value) was administered to antagonize any residual neuromuscular block when the frontal BIS score was $>70$. All patients were then extubated when the TOF values were $>90$. All patients were transferred to the PACU after the first postoperative neurological examination.

\section{Results}

The patients' demographic and surgical data are summarized in Table 1. There were significant correlations between the frontal and nasal BIS values at all time
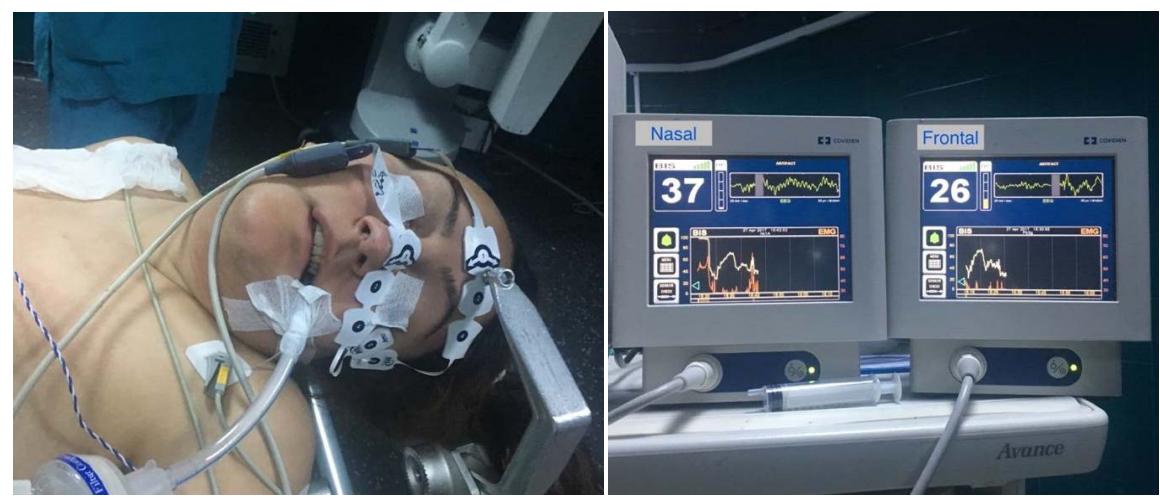

Figure 1. Nasal and fronatal BIS sensor placement. 
Table 1. Patient demographics.

\begin{tabular}{cc}
\hline Age $(\mathrm{yr})$ & $46.25 \pm 15.36$ \\
Gender $(\mathrm{F} / \mathrm{M})$ & $28 / 34$ \\
ASA I-II & $29 / 33$ \\
Height $(\mathrm{cm})$ & $165.49 \pm 8.17$ \\
Weight $(\mathrm{kg})$ & $64.80 \pm 11.53$ \\
BMI $\left(\mathrm{kg} / \mathrm{m}^{2}\right)$ & $23.49 \pm 3.05$ \\
Operation $(\mathrm{n}: 62)$ & Spinal (31) and cranial (31) \\
\hline
\end{tabular}

points. At the same time, according to the correlation coefficient, correlation was stronger during induction and awakening (Table 2, Figure 2, Figure 3). None of the patient interviews indicated awareness. One patient reported dreaming.

\section{Discussion}

The nasal BIS values were significantly correlated with the gold standard frontal BIS values at all time points. The difference between BIS values obtained from the forehead and nasal areas were considered identical, especially at the beginning-induction phase and during the awakening, emergence and early recovery period. Correlation was the similar during the maintenance period. The ICC was 0.78 indicating a good correlation between frontal and nasal sensors.

EEG activity is not homogeneous across the scalp even in normal awake or anaesthetized patients. Thus, the lack of EEG homogeneity in some clinical situations-including the artifact-free conditions of the present study-is not particularly surprising. The ability of the BIS algorithm such as other EEG-signal treatments to identify these local variations is of interest for potential clinical applications [6].

A few studies [6] [7] [8] have proposed that the BIS score is a topographic-dependent variable in light of the heterogeneous EEG findings in BIS sensors placed on non-frontal areas. Lee et al. compared frontal BIS monitoring with mandibular electrode position and reported strong correlations between frontal and mandibular sensor placement [6]. Unlike the frontal or occipital area, no EEG is generated under the mandible and thus detectable EEG or BIS is likely conducted from other parts of cerebral cortex. The exact locations remain unknown and warrant further study.

Another study [8] compared occipital and frontal placements. Here, occipital placement showed a +10 BIS score bias under deep anesthesia and a -10 BIS bias before induction. Although the nature of the BIS algorithm is proprietary, this result may be due to the predominance of the posterior alpha $(\alpha)$ waves in the awake brain and the generation of delta $(\delta)$ and theta $(\theta)$ activity under deep propofol anesthesia. In another study, Shiraishi et al. [9] compared BIS values obtained from frontal and occipital areas during propofol/fentanyl anesthesia. The BIS values in this study showed a strong correlation between frontal and 
Table 2. ICC and 95\% CI for pointwise and multiple repeated measurements.

\begin{tabular}{cc}
\hline Time & ICC $(95 \% \mathrm{CI})$ \\
\hline Premedication & $0.731(0.685-0.776)$ \\
Intubation & $0.424(0.376-0.4726)$ \\
Intraoperative & $0.771(0.62-0.862)$ \\
Extubation & $0.805(0.677-0.883)$ \\
PACU & $0.620(0.369-0.771)$ \\
\hline
\end{tabular}

ICC: Intraclass correlation coefficients, CI: confidence intervals.
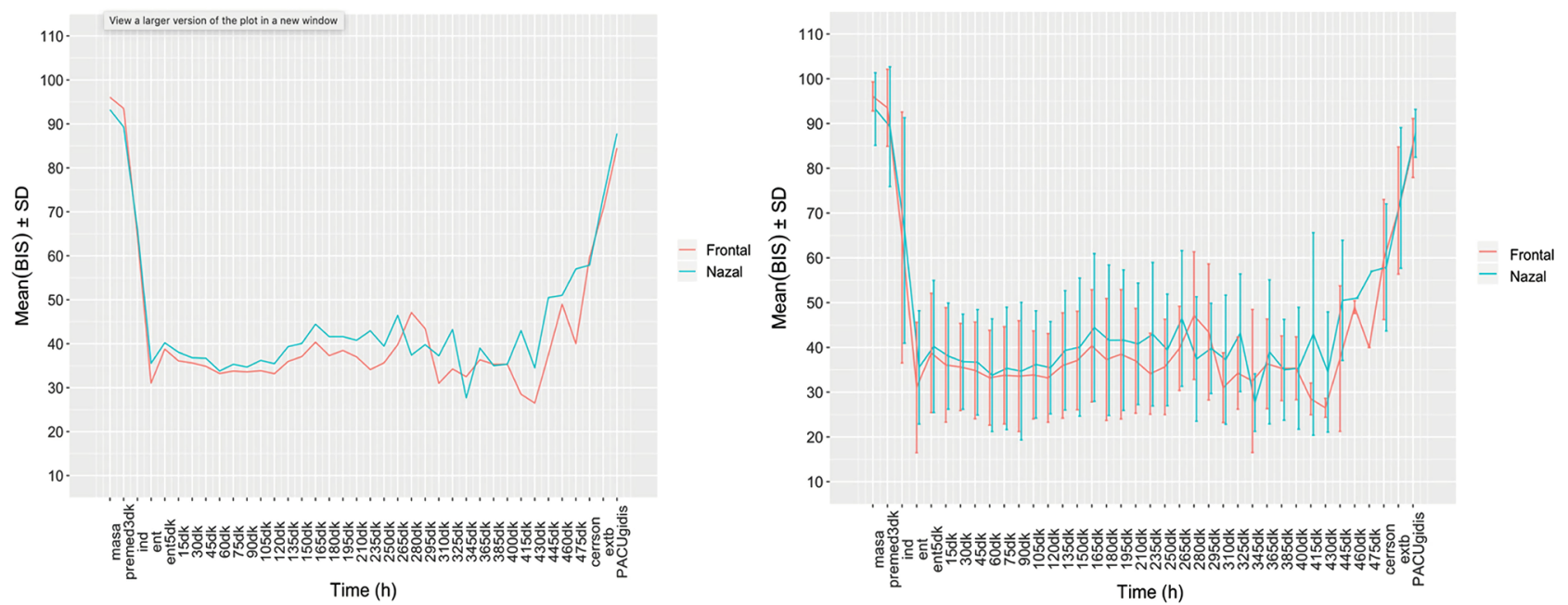

Figure 2. Relationship between nasal and frontal BIS measurements.

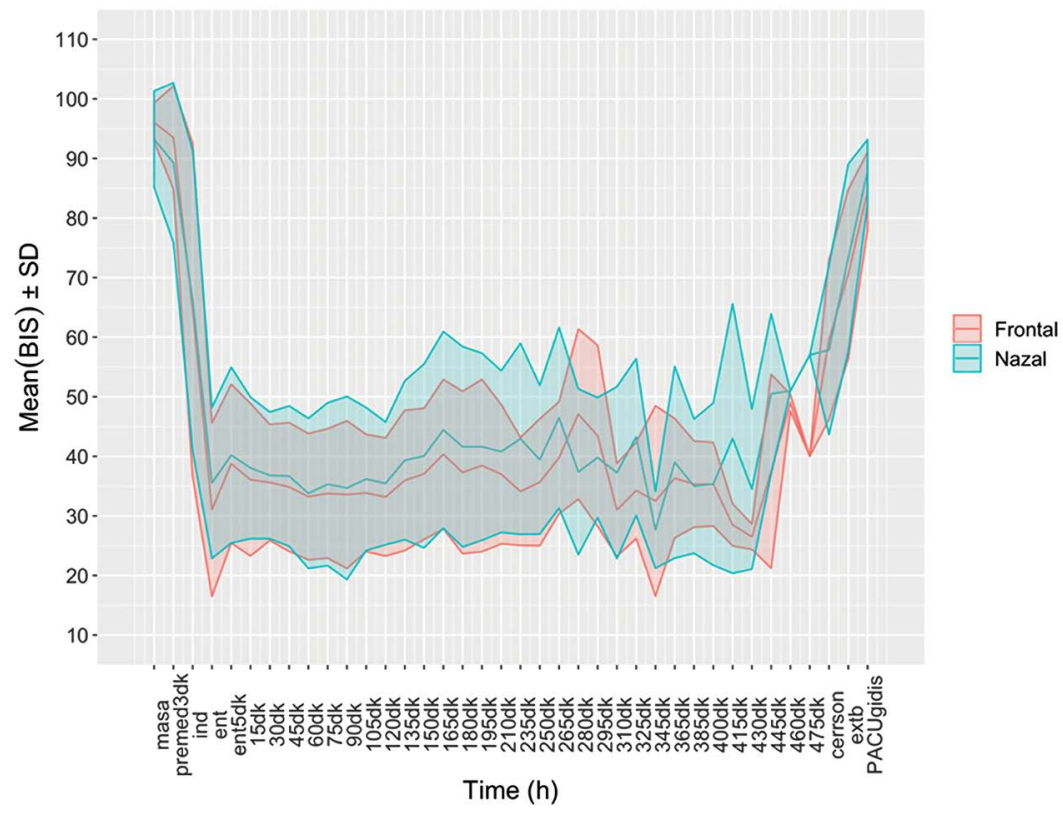

Figure 3. Relationship between nasal and frontal BIS measurements.

occipital montages $(\mathrm{r}(2)=0.96 ; \mathrm{P}=0.03)$. However, this study had weak correlation between two positions during awakening $(r=0.391)$ and LOC $(r=0.341)$ 
time points when no correlation at all was detected during emergence time point. These results are different from ours and show the significant correlation between nasal and frontal BIS values during all time points.

In neurosurgery, the recommended placement of electrodes for monitoring depth of anesthesia during surgery sometimes conflicts with the surgical site or patient positioning. Therefore, we conducted this study to evaluate the agreement and correlation of BIS values recorded from the standard frontal area as well as alternative nasal areas in neurosurgery patients. The nasal location of BIS electrodes has been previously demonstrated to be better in terms of correlation and application than occipital positioning, which is often of extreme usefulness for neurosurgical cases.

One limitation of our study is that we did not exclude drugs that could lead to false BIS values. Furthermore, BIS-VISTA does not generate raw EEG tracing for analysis, and thus we could not confirm whether the actual EEG recordings were identical. Although the BIS algorithm has not been formally validated, actual EEG recordings at each electrode on the frontal and nasal positions help confirm the accuracy and characteristics of the EEG signal arising from the nasal dorsum.

\section{Conclusion}

Our data reveal that BIS sensor placement on the nasal dorsum has comparable efficiency as standard front placement for measuring anesthesia depth (ICC 0.78 , $\mathrm{p}<0.001$ ), especially during the most variable periods of the surgery. This relationship is held regardless of the site of neurosurgical procedure (both cranial or vertebral). Thus, the nasal dorsum is a good and safe alternative when sensor positioning might interfere with the surgical site.

\section{Conflicts of Interest}

The authors have no conflicts of interest to declare.

\section{References}

[1] Mashour, G. and Avidan, M. (2015) Intraoperative Awareness: Controversies and Non-Controversies. British Journal of Anaesthesia, 115, i20-i26. https://doi.org/10.1093/bja/aev034

[2] Domino, K.B., Posner, K.L., Caplan, R.A. and Cheney, F.W. (1999) Awareness during Anesthesia: A Closed Claims Analysis. Anesthesiology, 90, 1053-1061. https://doi.org/10.1097/00000542-199904000-00019

[3] Lewis, S.R., Pritchard, M.W., Fawcett, L.J. and Punjasawadwong, Y. (2019) Bispectral Index for Improving Intraoperative Awareness and Early Postoperative Recovery in Adults. Cochrane Database of Systematic Review, 9, Article ID: CD003843. https://doi.org/10.1002/14651858.CD003843.pub4

[4] Medical Advisory Secretariat (2004) Bispectral Index Monitor: An Evidence Based Analysis. Ontario Health Technology Assessment Series, 4, 1-70.

[5] Koo, T.K. and Li, M.Y. (2016) A Guideline of Selecting and Reporting Intraclass 
Correlation Coefficients for Reliability Research. Journal of Chiropractic Medicine, 15, 155-163. https://doi.org/10.1016/j.jcm.2017.10.001

[6] Pandin, P., Van Cutsem, N., Tuna, T. and D’hollander, A. (2006) Bispectral Index Is a Topographically Dependent Variable in Patients Receiving Propofol Anaesthesia. British Journal of Anaesthesia, 97, 676-680. https://doi.org/10.1093/bja/ael235

[7] Lee, S. et al. (2004) Comparison of Bispectral Index Scores from the Standard Frontal Sensor Position with Those from an Alternative Mandibular Position. Anesthesia \& Analgesia, 98, 1773-1775.

[8] Dahaba, A. et al. (2006) BIS-Vista ${ }^{\text {Tm }}$ Occipital Montage in Patients Undergoing Neurosurgical Procedures during Propofol-Remifentanil Anesthesia. British Journal of Anaesthesia, 97, 676-680.

[9] Shiraishi, T., Uchino, H., Sagara, T. and Ishii, N. (2004) A Comparison of Frontal and Occipital Bispectral Index. Anesthesia \& Analgesia, 98, 1773-1775.

https://doi.org/10.1213/01.ANE.0000121344.69058.09 